\title{
Significance of abnormal pattern electroretinography in anterior visual pathway dysfunction
}

\author{
G E HOLDER
}

From the Regional Department of Clinical Neurophysiology, Brook General Hospital, Shooters Hill Road, London SE18 $4 L W$

SUMmARY The pattern electroretinogram (PERG) findings in 72 patients with disease of the anterior visual pathways are presented in relation to the site of dysfunction. Two components of the PERG are described which are selectively involved in different pathologies. In particular, the main positive P50 component is invariably affected in retinal/macular dysfunction, whereas the late negative $\mathrm{N} 95$ component is principally affected in optic nerve disease.

The pattern electroretinogram (PERG) is becoming increasingly used in the investigation of patients with anterior visual pathway disease, as it may enable a functional assessment of the retinal ganglion cells. Absent or reduced PERGs in the presence of intact flash ERGs have been described in three cases of optic neuritis, 'in a case of glaucoma, ${ }^{2}$ and in a patient with optic atrophy following traumatic optic nerve damage. ${ }^{3}$ These findings were in keeping with the experimental work of Maffei and Fiorentini, ${ }^{4}$ subsequently confirmed by Hollander et al., ${ }^{5}$ who had demonstrated that the PERG in the cat gradually disappeared over a four-month period following optic nerve section while the flash ERG was essentially unaffected. Histological examination at that time showed degeneration of the ganglion cells with minimal preganglionic damage. These results have been taken to indicate that the ganglion cell layer is implicated in the generation of the PERG, whereas it is known that the conventional flash ERG arises in the receptor and inner nuclear layers of the retina.

More detailed reports of PERGs in optic nerve or macular dysfunction have subsequently appeared, ${ }^{6-15}$ but there has been disagreement among these authors as to whether the PERG is consistently affected in optic nerve disorders. Indeed some authors $^{1617}$ have concluded that the PERG has no or little value in the assessment of patients with suspected optic nerve disease. Similarly, no definite conclusions as to the site of PERG origin have been reached.

This report reviews the PERG findings in 72 patients in whom the PERG was abnormal and in

Correspondence to Dr G E Holder. whom a diagnosis has been established. The results are presented in an attempt to elucidate further the possible diagnostic role of the PERG and to examine whether there may be specific PERG changes in particular disease processes. Preliminary accounts of these findings have been presented. ${ }^{18} 19$

\section{Materials and methods}

The checkerboard PERG was recorded by our standard techniques. ${ }^{20}$ Gold foil recording electrodes (Carter and Hogg, London) were placed in the fornix of the lower eyelid and referred to ipsilateral outer canthus silver-silver chloride electrodes with a mid forehead earth. Ipsilateral temple electrodes have been shown to minimise any possible contamination from the PVEP. ${ }^{21}$ The signals were processed by a Nicolet CA1000 averaging computer with internal artefact rejection and a bandwidth of $1-100 \mathrm{~Hz}-3 \mathrm{~dB}$. There were a minimum of 200 averages per trial with a minimum of two trials performed to confirm the reproducibility of the waveform. Checkerboard pattern reversal stimulation was performed by a Digitimer moving mirror stimulator subtending a total field of $14^{\circ}$ at the eye with an individual check size of $34^{\prime}$. Mean luminance was $400 \mathrm{~cd} / \mathrm{m}^{2}$ with $89 \%$ contrast. Stimulation rate was $4 \cdot 8$ reversals/s, with an analysis time of $200 \mathrm{~ms}$. (In some earlier recordings a rate of 6 reversals/s with an analysis time of $100 \mathrm{~ms}$ was used.) Binocular stimulation was performed, spectacle correction being used where appropriate. The advantage of binocular registration of the PERG is that, in patients with a unilateral reduction in visual acuity, fixation is maintained by the eye in which acuity is preserved. 
It is our experience that blink artefact can be a major problem in obtaining consistent recordings even when using the integral artefact reject system of the averager. We therefore use a technique of 'interrupted stimulation' which we find effective in minimising this type of artefact. The patient is instructed to look at the central fixation spot and to try not to blink while the pattern is moving. Every 4-6 s stimulation is interrupted, the patient told to blink a few times, and stimulation then resumed. We find this technique to be much more effective in obtaining waveforms of low variability than purely relying on the artefact reject system of the averager.

The patients described form part of a larger population routinely referred for electrodiagnostic assessment, mostly by neurologists, neurosurgeons, or ophthalmologists and have been selected for this study only because they had abnormal PERGs.

The normal control group consisted of volunteer members of the hospital staff with no significant ophthalmic or neurological history other than possible corrected refractive error.

\section{Results}

The normal checkerboard PERG recorded under our standard conditions consists of a prominent positive component at $52 \mathrm{~ms}$ (P50) followed by a larger negative component at some $93 \mathrm{~ms}$ (N95). In some patients a small early negative component at some 35 $\mathrm{ms}$ is also present. There is a superficial similarity between this waveform and the flash ERG which has led some authors to use the term 'b-wave' to describe the main positive component of the PERG. ${ }^{11-22} \mathrm{We}$ have not adopted this terminology because it is unlikely that these components represent the same physiological processes. We merely describe the components by their polarity and approximate mean latency. If it is thought that this may lead to confusion with components of the pattern visual evoked potential (PVEP), perhaps the terms $\alpha, \beta$, and $\gamma$ could be used to avoid confusion with flash ERG

Table 1 PERG normal values (16 subjects, 18-48 years, mean age 27.4 years)

\begin{tabular}{llll}
\hline Latency $(\mathrm{ms})$ & Range & Mean & $S D$ \\
\hline P50 & $48-55$ & $51 \cdot 67$ & $2 \cdot 25$ \\
N95 & $87-100$ & $93 \cdot 11$ & 3.48 \\
Amplitude $(\mu \mathrm{V})$ & & & \\
P50 & $2 \cdot 5-5 \cdot 0$ & $3 \cdot 74$ & 0.81 \\
N95 & $3 \cdot 6-8 \cdot 0$ & $5 \cdot 71$ & $1 \cdot 15$ \\
N95:P50 & $1 \cdot 25-2 \cdot 0$ & 1.54 & 0.18 \\
Interocular ratios & & & \\
P50 & $0 \cdot 88-0.97$ & 0.93 & 0.04 \\
N95 & $0 \cdot 83-0.98$ & 0.92 & 0.05 \\
N95:P50 & $0 \cdot 85-0.96$ & 0.93 & 0.03 \\
\hline
\end{tabular}

components. ${ }^{23}$ The ' $p$ ', ' $q$ ', and ' $r$ ' terminology of Kirkham and Coupland ${ }^{24}$ is not thought to be appropriate, as these terms have long established uses in electrocardiography.

Analysis of the PERG concentrated on the latency of P50, and the peak P50 and N95 amplitudes as measured from their points of onset. The intraocular N95:P50 ratio and the interocular ratios were also calculated.

Normal values are shown in Table 1. Of particular interest is the relatively high degree of interocular symmetry (shown by the low variability of the interocular ratios). This confirms the results of Arden et al. ${ }^{25}$ but is in disagreement with the high variability found by Kirkham and Coupland..$^{26}$ Although we consider that the relatively low variability of our findings is at least in part due to our recording technique using 'interrupted stimulation' (see above), we do not have an explanation for this apparent discrepancy between the findings of other authors. An important feature of our waveform analysis is the use of the intraocular N95:P50 amplitude ratio. This stable measure can be particularly useful in the assessment of patients with bilateral disease (see Fig. 3).

The results in patients are shown in Table 2 . The criteria for abnormality were set at 2.5 standard deviations from mean values. In most cases the PERG changes were restricted to amplitude. In two patients, however, one with a central retinal vein occlusion, and one with a retinal detachment, the P50 latency was increased. It is of particular interest that two patients with optic nerve disease showed an abnormality confined to the N95 component when first examined but had developed a P50 abnormality at follow-up.

Table 2 Pattern ERG findings in 72 patients with anterior visual pathway dysfunction

\begin{tabular}{|c|c|c|c|}
\hline & \multirow{2}{*}{$\begin{array}{l}\text { No. of } \\
\text { patients }\end{array}$} & \multicolumn{2}{|c|}{ PERGabnormality } \\
\hline & & $P 50 \pm N 95$ & N95 only \\
\hline $\begin{array}{l}\text { Retinal disease, e.g., retinitis } \\
\text { pigmentosa, retinal vascular } \\
\text { lesions, maculopathy, cone } \\
\text { dystrophy, etc. }\end{array}$ & 28 & 28 & 0 \\
\hline $\begin{array}{l}\text { Reduced acuity due to cataract } \\
\text { Optic nerve disease, e.g., } \\
\text { demyelination, compression, } \\
\text { ischaemic optic neuropathy, etc. }\end{array}$ & $36^{*}$ & $9^{*}$ & $29^{*}$ \\
\hline Glaucoma & 3 & 3 & 0 \\
\hline Amblyopia & 2 & 2 & 0 \\
\hline Congenital optic disc abnormality & 1 & $\mathbf{0}$ & 1 \\
\hline
\end{tabular}

*Two patients with optic nerve demyelination initially showed an amplitude reduction confined to $\mathrm{N} 95$, but subsequent follow-up examination revealed the development of a P50 abnormality. 
Illustrative examples of the findings in patients with retinal and optic nerve disease are shown in Figs. 1-3. All PERGs are the superimposition of two trials to demonstrate the reproducibility of the waveform. The patient with the maculopathy was selected because the PERG from the affected eye is present but reduced. In the patients with severe pigmentary retinopathy and central retinal involvement, in cone dystrophies, senile disciform macular degeneration, etc., the PERG was usually absent from affected eyes. In no case of optic nerve disease, including one eye with 'no perception of light' due to optic nerve compression from a pituitary tumour, was the PERG completely absent.

\section{Discussion}

The PERG abnormalities of the patients in our study with generalised retinal or macular dysfunction occur in the main positive (P50) component. This is in agreement with the findings of previous authors. ${ }^{9427}$ Other reports of PERG abnormalities in macular disease have also appeared ${ }^{728}$ but the authors used steady state stimulation, which precludes individual
Fig. 1 Pattern ERG findings in a patient aged 20 with a right maculopathy. The P50 component is of significantly reduced amplitude in the recordings from the right eye. Calibration $3 \mu \mathrm{V}, 40 \mathrm{~ms}$.
VOD $6 / 12$, VOS

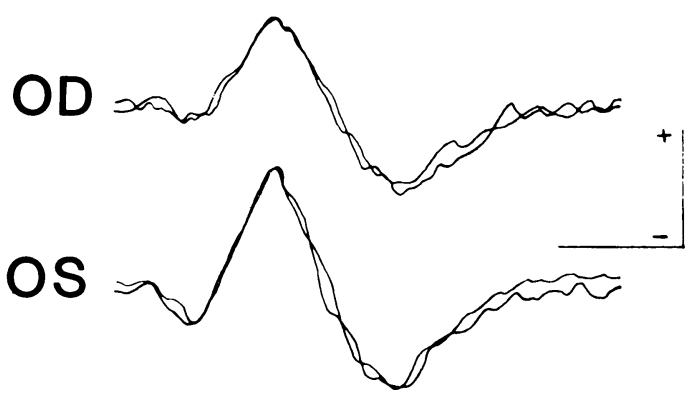

VOD $6 / 5$, VOS $6 / 5$

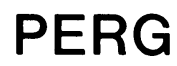

Fig. 2 Pattern ERG findings in a patient aged 39 with multiple sclerosis and right retrobulbar neuritis. The N95 component is of significantly reduced amplitude in the recordings from the right eye, the P50 component being unaffected. Calibration $3 \mu \mathrm{V}$, $40 \mathrm{~ms}$.

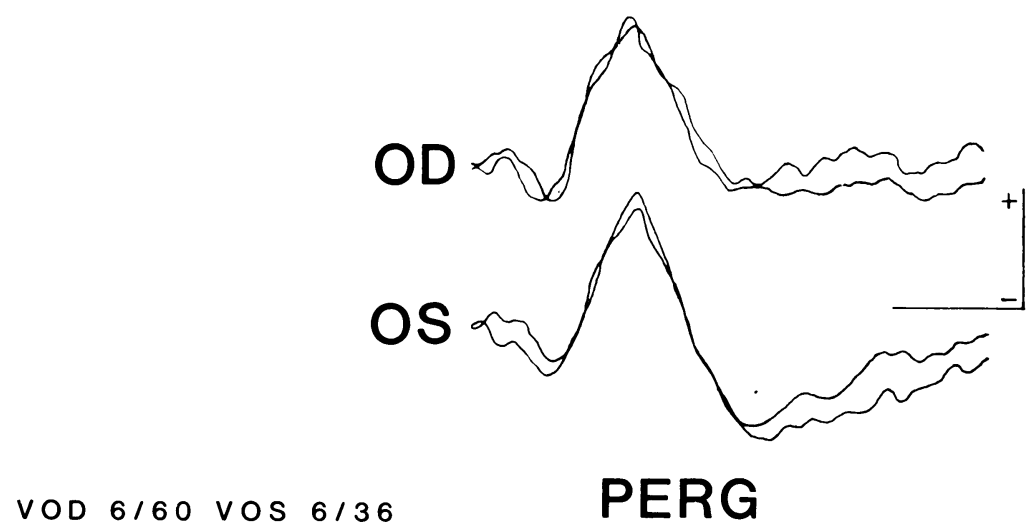

Fig. 3 Pattern ERG findings in a patient aged 64 with bilateral idiopathic optic atrophy. The N95 component, as reflected in the N95:P50 intraocular ratio, is bilaterally subnormal. Calibration $3 \mu \mathrm{V}, \mathbf{4 0} \mathrm{ms}$.

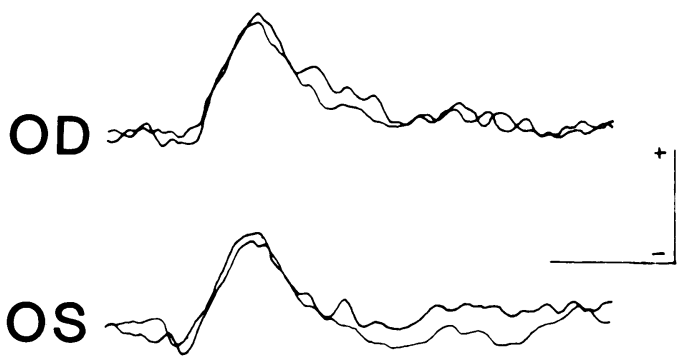


P50 and N95 assessment. It is similarly difficult to compare the present findings in optic nerve disease with previous reports from authors using steady state stimulation. ${ }^{7815}$ Other authors ${ }^{6101316}$ have examined the P50 component of the transient PERG and described abnormalities of this component associated with optic nerve dysfunction. Although abnormalities of the P50 component occurred in some of our patients, the predominant abnormality $(81 \%)$ we observed in patients with abnormal PERGs and optic nerve disease was involvement of the N95 component, the P50 component being spared. In addition, although absent PERGs were often seen in patients with retinal dysfunction, no patient with optic nerve disease had an absent PERG.

Indeed, the most important feature of our results is the selective effect of different pathological conditions on the two PERG components studied. In no patient in our series with retinal or macular disease was an abnormality confined to the N95 component; the P50 component was involved in all patients with abnormal PERGs and retinal dysfunction. In contrast, $81 \%$ of our patients with optic neuropathies and abnormal PERGs showed an abnormality confined to the N95 component. Porciatti and von Berger ${ }^{11}$ have previously noted that in some patients with optic nerve disease the N95 component may be affected, but unfortunately they supplied no further details. We have been unable to trace any other clinical report including analysis of this component. Our findings establish that the N95 component is the more sensitive indicator of optic nerve dysfunction and also suggest that failure to examine this component may result in false negative examinations. This may explain some of the discrepancies between previous authors as to the incidence of PERG abnormalities in optic neuropathies: abnormalities of the P50 component to which other authors have restricted their attention occurred in only $19 \%$ of our patients with optic nerve dysfunction and abnormal PERGs. Our findings also appear to refute the conclusions of Kirkham and Coupland ${ }^{16}$ and Ota and Miyake $^{17}$ that the PERG has little value in the assessment of optic nerve disease.

The results also readily lead to speculation as to the site or sites of origin of the PERG. Berninger and Schuurmans ${ }^{21}$ have reported that the late negative component of the checkerboard PERG (our N95) exhibits spatial tuning in normal subjects, concluding that the retinal ganglion cell layer is therefore involved in its generation. Similar conclusions have also been drawn for the second harmonic component of the steady state PERG. ${ }^{14}$ Our results in patients with optic nerve disease and abnormal PERGs provide clinical evidence to support the theory that the N95 component of the PERG is generated in the proximal retina, presumably the ganglion cells, which would be expected, at least in the fullness of time, to show abnormalities due to retrograde degeneration.

It is important here, particularly if the PERG is to play a significant part in the diagnostic armamentarium, to ensure that the PERG, particularly the N95 component, is independent of the cortically generated visual evoked potential (VEP). Berninger and Schuurmans ${ }^{21}$ extensively investigated the effects of different reference electrodes and concluded that, if an ipsilateral temple reference (similar to that used in our study) is used, there is no doubt that both the P50 and the N95 components are generated in the retina and do not reflect contamination from the VEP. Plant $e t$ al. ${ }^{15}$ reached similar conclusions for the steady state PERG. Our own observations in patients lead to similar conclusions when pattern VEP data are considered (in preparation). Examples illustrating this dissociation between the PERG and the pattern VEP are shown in Fig. 4.

It is more difficult to draw conclusions as to the site of origin of the P50 component from our data. This component was affected in all our patients with macular or generalised retinal dysfunction, which would be expected to primarily involve distal retinal layers in patients with glaucoma and in patients with reduced visual acuity due to cataract. The selective involvement of the P50 and N95 components in different pathological states does, however, suggest that they are generated in different retinal layers, in keeping with the suggestion of Berninger and Schuurmans. ${ }^{21}$ These authors ${ }^{30}$ have also suggested, using low stimulus luminous levels, that the main positive component of the PERG is luminance related, whereas the late negative component is contrast related. This observation resulted in the conclusion that the late negative component is probably generated in the ganglion cells, the main positive component being generated more distally.

In conclusion, the N95 component of the PERG is selectively affected in $81 \%$ of the patients studied with definite optic nerve disease and abnormal PERGs, invovement of the P50 component only occurring in $19 \%$ of the cases examined. The P50 component, however, was invariably affected in retinal disease, in glaucoma and in reduced visual acuity due to cataract, no such patient having a PERG abnormality confined to the N95 component. In addition, delayed PERGs were seen only in retinal dysfunction, and in one of those patients a pattern VEP delay of similar magnitude was observed. It has previously been reported ${ }^{31}$ that retinal dysfunction can give rise to delayed pattern VEPs, even in the presence of normal visual acuity, and it is now standard practice in our clinic to record the PERGs in 
PERG

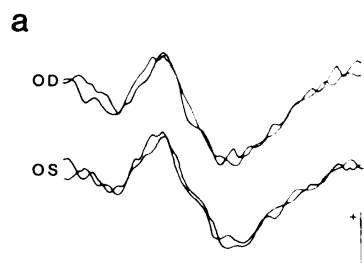

b

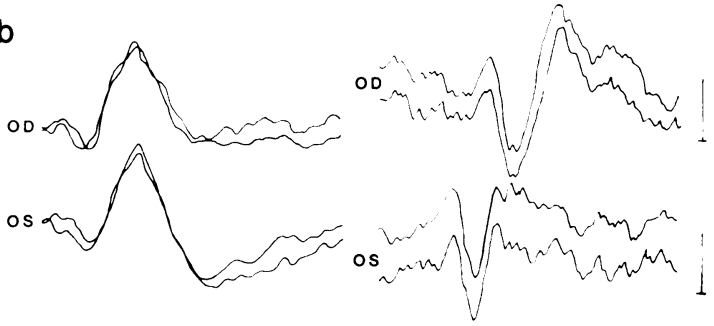

C

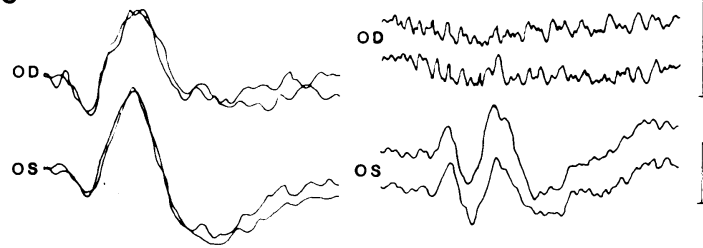

Fig. 4 Pattern VEP and pattern ERG (PERG) findings in three patients with optic nerve demylination which demonstrate the independence of the N95 component of the PERG and the pattern VEP. (a) A patient with a unilateral pattern VEP delay but normal, symmetrical PERGs. (b) $A$ patient with a unilateral pattern VEP delay with a unilateral $N 95$ amplitude reduction in the PERG from the affected eye. (c) A patient with a unilateral pattern VEP extinction and $a$ unilateral N95 amplitude component reduction in the PERG. PERG traces are the superimposition of two trials to demonstrate the reliability of the waveform. Pattern VEPs are recorded from the right (upper trace) and left hemispheres in response to monocular stimulation of each eye in accordance with our standard techniques. ${ }^{29}$ Calibration for all PERGs $3 \mu V, 40 \mathrm{~ms}$. VEP analysis time $320 \mathrm{~ms}$, amplitude calibration $5 \mu \mathrm{V}$.

any patient with a delayed VEP. Certainly the diagnostic indicators detailed in the present report confirm the role of the PERG in the assessment of patients with anterior visual dysfunction, and it is therefore suggested that PERG recording should be a routine part of an ophthalmic electrodiagnostic protocol, particularly if there is a suspicion of optic nerve disease.

I am indebted to Dr Gordon Plant for access to material in press, to Mr John Shilling for his comments on the original version of this manuscript, and to the Medical Photography Department, Greenwich District Hospital, for assistance with the figures.

\section{References}

1 Fiorentini A, Maffei L, Pirchio M, Spinelli D, Porciatti V. The ERG in response to alternating gratings in patients with diseases of the peripheral visual pathway. Invest Ophthalmol Vis Sci 1981; 21: $490-3$.

2 May JG, Ralston JV, Reed JL, van Dyk HJL. Loss in patternelicited electroretinograms in optic nerve dysfunction. $A m J$ Ophthalmol 1982; 93: 418-22.

3 Dawson W, Maida T, Rubin M. Human pattern evoked retinal responses are altered by optic atrophy. Invest Ophthalmol Vis Sci 1982; 22: 796-803.

4 Maffei L, Fiorentini A. Electroretinographic responses to alternating gratings before and after section of the optic nerve. Science 1981; 211: 953-55.

5 Hollander H, Bisti S, Maffei L, Hebel R. Electroretinographic responses and retrograde changes of retinal morphology after intracranial optic nerve section. A quantitative analysis in the cat. Exp Brain Res 1984; 55: 483-93.

6 Arden GB, Vaegan, Hogg CR. Clinical and experimental evidence that the pattern electroretinogram (PERG) is generated in more proximal retinal layers than the focal electroretinogram (FERG). Ann NY Acad Sci 1982; 388: 580-601.

7 Sherman J. Simultaneous pattern reversal electroretinograms and visual evoked potentials in diseases of the macula and optic nerve. Ann NY Acad Sci 1982; 388: 214-26.

8 Bobak P, Bodis-Wollner I, Harnois C, et al. Pattern electroretinograms and visual evoked potentials in glaucoma and multiple sclerosis. Am J Ophthalmol 1983; 96: 72-83.

9 Arden GB, Carter RM, Macfarlan A. Pattern and ganzfeld electroretinograms in macular disease. Br J Ophthalmol 1984; 68: $878-84$

10 Persson HE, Wanger P. Pattern reversal electroretinograms and visual evoked cortical potentials in multiple sclerosis. $\mathrm{Br} \mathrm{J}$ Ophthhalmol 1984; 68: 760-4.

11 Porciatti V, von Berger GP. Pattern electroretinogram and visual evoked potential in optic nerve disease: early diagnosis and prognosis. Doc Ophthalmol Proc Ser 1984; 40: 117-26.

12 Porciatti V, von Berger GP. Retinal and cortical evoked potentials in response to alternating gratings in the diagnosis of optic nerve diseases. In: Nodar RH, Barber C, eds. Evoked Potentials II. London: Butterworths, 1984; 319-28.

13 Serra G, Carreras M, Tugnoli V, Manca M, Cristofori MC. Pattern electroretinogram in multiple sclerosis. J Neurol Neurosurg Psychiatry 1984; 47: 879-83.

14 Plant GT, Hess RF. The electrophysiological assessment of optic neuritis. In: Hess RF, Plant GT, eds. Optic neuritis. London: Cambridge University Press 1986; 192-229.

15 Plant GT, Hess RF, Thomas SJ. The pattern evoked electroretinogram in optic ncuritis: a combined psychophysical and electrophysiological study. Brain 1986; 109: 469-90.

16 Kirkham TH, Coupland SG. The pattern electroretinogram in optic nerve demylination. Can J Neurol Sci 1983; 10: 256-60.

17 Ota I, Miyake Y. The pattern electroretinogram in patients with optic nerve disease. Doc Ophthalmol 1986; 62: 53-60.

18 Holder GE. Pattern ERG abnormalities in anterior visual pathway disease. Electroencephalogr Clin Neurophysiol 1985; 61: S135.

19 Holder GE. The importance of the N95 component of the pattern ERG in the assessment of anterior visual pathway dysfunction. Abstracts XXIV Symposium of the International Society for Clinical Electrophysiology of Vision, Palermo, May 1986.

20 Holder GE, Huber MJE. The effects of miosis on pattern and flash electroretinogram and pattern visual evoked potential. Doc Ophthalmol Proc Ser 1984; 40: 109-16.

21 Berninger T, Schuurmans RP. Spatial tuning of the pattern ERG across temporal frequency. Doc Ophthalmol 1985; 61: 17-25.

22 Korth M. Human fast retinal potentials and the spatial properties of a visual stimulus. Vision Res 1981; 21: 627-30. 
23 Bodis-Wollner I. In discussion of Holder GE. ${ }^{18}$

24 Kirkham TH, Coupland SG. Abnormal pattern electroretinograms with macular cherry-red spots: evidence for selective ganglion cell damage. Curr Eye Res 1981; 1: 367-72.

25 Arden GB, Carter RM, Hogg C, Siegel IM, Margolis S. A gold foil electrode: extending the horizons for clinical electroretinography. Invest Ophthalmol Vis Sci 1979; 18: 421-6.

26 Kirkham TH, Coupland SG. Pattern ERGs and check size: absence of spatial frequency tuning. Curr Eye Res 1983; 2: 511-21.

27 Wanger P, Persson HE. Pattern reversal electroretinograms in unilateral glaucoma. Invest Ophthalmol Vis Sci 1983; 24: 749-53.
28 Sokol S, Bloom BH. Macular ERGs elicited by checkerboard pattern stimuli Doc Ophthalmol 1977; 13: 299-305.

29 Holder GE. The effects of chiasmal compression on the pattern visual evoked potential Electroencephalogr Clin Neurophysiol $1978 ; 45: 278-80$.

30 Schuurmans RP, Berninger T. Luminance and contrast responses recorded in man and cat. Doc Ophthalmol 1985; 59: 187-97.

31 Holder GE, Chesterton JR. The visual evoked potential in Harada's disease. Neuroophthalmology 1984; 4: 43-5.

Accepted for publication 8 July 1986. 